\title{
Verkündigung und Praxis Vorüberlegungen zum Verhältnis von Verkündigung und Werbung
} von Ottmar John

\section{Werbung als Vervollkommnung der kirchlichen Verkündigung?}

Seit über 100 Jahren gehen Pfarrer, Theologen und sonstige Verantwortliche mit der Frage um, ob die Kirche sich der Werbung bedienen solle. ${ }^{1}$ In positiven Stellungnahmen wird der Einsatz von Werbung und Marketing als lineare Erweiterung der kirchlichen Verkündigung aufgefaßt. Werbung erscheint dann als Erfüllung des biblischen Missionsbefehls, das Evangelium bis an die Enden der Welt zu tragen. Werbung und massenmediale Kommunikation sind dabei eine weitere Etappe im Universalisierungsvorgang des Christentums; diese Universalisierung ist nicht nur eine Auslegung der geoffenbarten Wahrheit in der Kategorialität griechischer Philosophie (Hellenisierung), nicht nur eine geographische Ausdehnung (von der Germanen- bis zur Indianermisssion), sondern in der Moderne heißt Universalisierung des Christentums, eine höchstmögliche Vielfalt der Formen und Mittel zu erreichen. Signifikant für den jüngsten, hier zur Debatte stehenden Universalisierungsschub ist dann die Befreiung der Verkündigung aus den Fesseln der monologischen, liturgisch fixierten, an Amtsträger gebundenen Kanzelpredigt; sie wird erweitert durch das Gespräch, den Unterricht, die Kunst, durch „Passantenpastoral“2 bis hin zur Radiound Fernsehverkündigung. ${ }^{3}$ "Im Zeitalter der Technik wird man sich aller Mittel bedienen, die Gott uns zur Verfügung stellt, auch der technischen Mittel. Auch durch sie kann Verkündigung geschehen, und zugleich Werbung für die Kirche."4

Dr. theol. Ottmar Johan ist Habilitand an der Katholisch-Theologischen Fakultät der Universität Münster.

1 Siehe dazu den instruktiven geschichtlichen Überblick von St. W. Hillebrecht, Kirchliche Werbung. Ein Abriß ihrer Geschichte und Problematik, in: CS 3/95, 228-252, 230.

2 In den Umkreis derartiger pastoraler Versuche gehören auch die schon älteren 10-Minuten-Andachten, mit denen Citygemeinden den Kommunikationsformen passierender, vorbeieilender Großstadtbewohner und vor allem deren Zeitstruktur gerecht werden wollen.

3 E. Bieger/W. Fischer u.a. ( $\mathrm{Hg}$.), zeitgeistlich. Religion und Fernsehen in den neunziger Jahren, Köln 1993.

4 C. Pohlmann, Formen heutiger Verkündigung, in: Handbuch der Verkündigung (hg.v. B. Dreher, N. Greinacher u.a.) Freiburg 1970, Bd. II, 179-208, 206 (im Folgenden $\mathrm{HbV}+\mathrm{Band}+$ Seite). Der ganze Artikel bietet einen guten 
Unabhängig davon bietet sich die Werbeindustrie der Kirche zur Behebung von deren offensichtlicher Akzeptanzkrise an. Dieses Angebot bezieht sich entweder auf die Institutionskrise ${ }^{5}$ oder auf die "Predigtnot" und das wachsende Unverständnis gegenüber der Verkündigung. ${ }^{6}$ Dabei braucht nicht weiter auf das Problem eingegangen zu werden, ob die Kirche sich selbst darstellen und für sich selbst werben könne.7 Wenn man darüber nachdenkt, was passiert, wenn die Kirche sich zu sich verhält - und das passiert in jeder Selbstdarstellung -, dann entdeckt man schnell einen eigentümlichen Zusammenhang von Nichtidentität und Reflexion. Die Kirche vollzieht sich, indem sie über sich hinausweist, auf Gott und das in ihm geschenkte Heil. Dieses Hinausweisen geschieht jedoch nicht direkt (intentio recta), sondern reflexiv in der Behauptung der "Herkünftigkeit" (Rahner) der Kirche aus dem Erlösungsgeschehen.

Eine Selbstdarstellung der Kirche als Institution würde demgegenüber eine Identität von darstellendem Subjekt und Inhalt mit sich bringen. Damit würde in der Imagewerbung die Kirche sich verfehlen. Denn sie ist nur sie selbst, wenn sie sich auf den Auferstandenen bezieht. In der Werbung würde sie sich selbst zu einer normalen und beliebigen gesellschaftlichen Institution säkularisieren.

Diese Einsicht in die Unmöglichkeit der Kirchenwerbung koinzidiert erstaunlicherweise mit empirischen Erhebungen, die das $\mathrm{Zu}$ schauerinteresse an Werbung und massenmedialen Produktionen untersuchen: Die "Kirche" als Institution trifft auf das denkbar geringste Interesse, Religion, ihre Sache, dagegen auf ein erheblich höheres.

Von daher liegt es nahe, daß nicht die Kirche sich selbst darstellt, sondern in den Massenmedien und in der Werbung ihren Auftrag zu vollziehen sucht: das Evangelium, den auferstandenen Christus zu verkünden. Macht sie dieses sachadäquat, wird mehr von ihrem Wesen deutlich als in einer Selbstdarstellung. Insofern ist die Kinche nicht zur Selbstdarstellung beauftragt, sondern zur Darstellung ihres Selbst, ihres

Überblick über die in den sechziger Jahren aufbrechende Vielfalt der Formen kirchlicher Verkündigung. Die kontinuierliche Reihung der Formen und Mittel vertritt auch F. Klostermann, Die Träger der Verkündigung, in: HbV 1, 363-408, 400f: Wenn der Ort der Verkündigung stärker als bisher durch den Hörer bestimmt wird, kommt es zwangsläufig zu einer Plurifizierung der Formen und Mittel.

5 Die Symptome der kirchlichen Institutionskrise reichen dabei vom Ursprung der Neuzeit, der konfessionellen Spaltung, bis hin zu gegenwärtigen Austrittswellen und Einbrüchen im Kirchensteueraufkommen.

6 H. Jacob, Theologie der Predigt. Zur Deutung der Wortverkündigung durch die neuere katholische Theologie, Essen 1969, 1.

7 Diese Frage hat niemand so scharf gestellt wie K. Barth, Quousque tandem, in: Zwischen den Zeiten 8 (1930) H.1, 1-8. 
ihr äußerlichen Grundes, ihres unverfügbaren, absoluten Ursprunges. Insofern ist Verkündigung Selbstvollzug von Kirche. ${ }^{8}$ Sie verkündigt nicht sich, aber wenn sie den auch ihr vorgegebenen Offenbarungsinhalt verkündet, ist sie diesem Geschehen nicht nur äußerlich. Insofern kann gesagt werden, daß die Frage nach dem Verhältnis von Kirche und Werbung zu der fundamentaleren nach der Möglichkeit von Verkündigung durch Werbung führt.

Die Konvergenz von Verkündigung und Werbung erscheint also in hohem Maße plausibel; ja angesichts der Krise der Verkündigung scheint die Inanspruchnahme neuer Kommunikationsmittel und umfassender Marketingstrategien ein Gebot der Stunde zu sein. Diese Plausibilität soll im folgenden durch eine Reflexion auf die kirchliche Verkündigung befragt werden. Die Frage, ob die Krise durch Werbung überwunden werden kann, soll also durch die Beantwortung der Frage, was Verkündigung sei, angesetzt werden. Offen bleibt dabei zunächst die fast ebenso dringliche Frage, was Werbung sei und erst recht natürlich die Explizierung ihres Verhältnisses.

\section{Verkündigung und Offenbarung}

\subsection{Weltliche Nachrichtenübermittlung und kirchliche Verkündigung}

Obwohl "Verkündigung" heute ein theologischer und pastoraler Spezialbegriff ist und alltagssprachlich kaum Verwendung findet, entstammt das Wort nichtreligösen Zusammenhängen: Verkündigung ist die Handlung des Boten, der eine andernorts empfangene Nachricht mitteilt und öffentlich, rechtsverbindlich kundtut. ${ }^{9}$ Die vier beteiligten Grundelemente sind dabei unterschiedlich am Verkündigungsvorgang beteiligt: Bote, Nachricht und Empfänger sind direkt beteiligt; ihre raum-zeitliche Nähe zueinander ist Bedingung dafür, daß Verkündigung stattfinden kann. Der Geber der Botschaft ist dagegen nur indirekt beteiligt. Der Sinn der Verkündigung scheint darin zu bestehen, die raumzeitliche Differenz zwischen Empfänger und Geber zu überwinden; umso größer die Entfernung, desto notwendiger das Verkündigungsgeschehen.

Verkündigung ist in diesem Bild die Nahtstelle, die Begegnung zwischen Überbringer und Empfänger der Nachricht. Möglich ist die

8 Siehe dazu auch N. Greinacher, Verkündigung als Grundfunktion der Kirche, in: $\mathrm{HbV}$ I 335-362.

9 K. Lehmann/K. Rahner, Kerygma und Dogma, in: Mysterium salutis. Grundriß heilsgeschichtlicher Dogmatik (hg.v. J. Feiner, M. Löhrer), - im folgenden Mysal + Band + Seite - Bd.I, Einsiedeln/Zürich/Köln 1965, $622-707,623$. 
Verkündigung nur deswegen, weil ihr eine frühere Begegnung vorausgeht. Der Sender der Botschaft übergab diese dem Boten.

Man kann mit diesem Bild einen theologischen Verkündigungsbegriff von profanem Verkündigungsgeschehen unterscheiden: Dem profanen Verkündigungsgeschehen geht eine Übergabe der Botschaft voraus, die sich in ihrer Eigenart nicht von der Verkündigung unterscheidet. Das Verkündigungsgeschehen in der Glaubensgeschichte des Christentums folgt einem prinzipiell andersartigen Geschehen. Die Mitteilung des Senders an den Boten ist hier Offenbarungshandeln. Sein Stattfinden qualifiziert die grundsätzliche Besonderheit der Verkündigung und ermöglicht ihre Unterscheidung und Vergleichung mit natürlichen Verkündigungshandlungen. ${ }^{10}$

Kirchliche Verkündigung ist also in einem ersten Zugriff ein komplementärer Vorgang zur Offenbarung. ${ }^{11}$ Die erste Frage für die folgenden Überlegungen besteht also darin, was es für die Verkündigung der Kirche bedeutet, daß sie sich aus dem Offenbarungshandeln Gottes selbst herleitet und sich von ihm ermöglicht weiß.

\subsection{Der Grund der Verkündigung}

Der theologische Offenbarungsbegriff bezeichnet entweder einen Inhalt oder die Ubergabe dieser Inhalte, entweder, was Gott den Menschen sagt, oder die Art und Weise, wie er es sagt.

10 "Die Offenbarung ist die letzte Voraussetzung, Grund, Mitte und Norm für alles, was als christlich gelten will." W. Kasper, Offenbarung Gottes in der Geschichte, in: HbV I 53.

11 Dabei gibt es zwei wiederum komplementäre methodische Zugriffe: Erstens kann der Verkündigungsbegriff aus dem Offenbarungsgeschehen abgeleitet werden: Offenbarung muß verkündet werden, da sie a) vorgegeben ist, also niemand sie einfach so weiß und b) Offenbarung in der ununterbrochenen Annahme und Weitergabe erst wirklich und universal ist - siehe dazu W.Kasper, Offenbarung Gottes in der Geschichte, in: HbV I 70. Zweitens kann der Verkündigungsakt analysiert werden: Offenbarung zeigt sich dann als sein konstitutiver Inhalt, ohne den Verkündigung nicht sein könnte. Er ist in der Analyse als Bedingung erkennbar.

Die Analyse ist eigentlich die theologisch angemessenere Methode, denn es gibt eine theologische Reflexion der Offenbarung Gottes nur, weil sie in der Verkündigung tradiert ist. Nur unter der Bedingung, da $B$ die Offenbarung in der Geschichte weitererzählt worden ist, kann sie Reflexionsgegenstand sein. Jedoch müßte in diesem Verfahren mit einer Empirie der kirchlichen Verkündigungswirklichkeit angefangen werden. Es müßte angesetzt werden mit der Verkündigung, so wie sie in der Kirche wirklich von lebendigen Subjekten vollzogen wird, um ihre konstitutiven Elemente und Bedingungen, die erfüllt sein müssen, damit sie ihren Anspruch zurecht erheben kann, namhaft machen zu können. Da dieses hier aus Platzgründen nicht möglich ist, wird die Deduktion des Verkündigungsbegriffs aus dem der Offenbarung angewandt - sozusagen als methodische Verkürzung. 
Wenn Offenbarung vorrangig als Was, als Inhalt verstanden wird, dann ist das nicht eine beliebige Form, sondern bereits ein Vorbegriff dieses Inhaltes selbst: Mit ihm sagt Gott etwas über sich. In der Neuscholastik wurde Offenbarung in diesem Sinne als Mitteilung Gottes über sich verstanden. $\mathrm{Da}$ es sich um Offenbarungen handelte, konnte man dann im intellektuellen Nachvollzug einsehen: Die mitgeteilten Inhalte handelten über das innere Leben Gottes, seine Einfachheit und Trinität, die Einheit und Verschiedenheit von Menschlichkeit und Göttlichkeit des Erlösers etc. Diese Inhalte konnte der Mensch nicht von sich aus wissen, sie mußten ihm also von Gott mitgeteilt sein.

$\mathrm{Daß}$ der Mensch sie nicht von sich aus kennen kann, war daran ablesbar, daß sie sich entweder jedem konsistenten Begriff entzogen oder ihre intellektuelle Aneignung, ihr Nachvollzug die Vernunft in Widersprüche verstrickte. Zumindest kann aber die Vernunft wissen, $\mathrm{da} ß$ sie die Glaubensinhalte nicht vollständig begreifen und sich zu eigen machen kann - und darin ihre eigene Endlichkeit und Natürlichkeit reflektieren.

Wie konnten nun die Glaubensinhalte von beliebigen Verstandesrätseln oder Absurditäten, von Phantasien und menschlichen Einbildungen unterschieden werden? Wie kann die bleibende Geheimnishaftigkeit eines Inhaltes das vernunftangemessenes Indiz für deren Herkünftigkeit aus einem Offenbarungsvorgang sein? Die Unbeantwortbarkeit dieser Frage mittels eines gegenständlichen Offenbarungsbegriffs führte dazu, daß die Frage nach der Offenbarung als Vorgang immer weiter in den Vordergrund rückte. Für die Neuscholastik stand fest, daß Gott die Inhalte mitgeteilt hat. Deswegen waren sie authentisch und glaubwürdig. Deswegen konnten sie in der "Lehrverkündigung" der Kirche den Gläubigen zu Annahme vorgelegt werden. Umgekehrt hatten diese die Pflicht, sie anzunehmen; diese so vorgelegten Inhalte anzunehmen war notwendig für die Erlangung des Heils.12

Nun ist aber die Sicherheit, daß Gott etwas oder auch alles über sein inneres Leben mitgeteilt hat, alles andere als historisch beweisbar; die natürliche Erkenntnis der bedeutungs- und inhaltsunabhängigen Tatsache der Offenbarung scheiterte. ${ }^{13}$ Anderseits wollte Gott erlösend

12 Die Priorität der Offenbarung als Inhalt zieht ein Verkündigungsverständnis nach sich, in dem die Glaubensinhalte durch eine autoritative, dazu bevollmächtigte Instanz, nämlich das Lehramt, den Gläubigen vorgelegt werden - siehe dazu M. J. Scheeben, Gesammelte Schriften III (hg.v. M. Grabmann) Freiburg 1948, $41 \mathrm{ff}$.

13 A. Kolping spricht von einer Aporie der klassischen fundamentaltheologischen Tatsachenbeweise hinsichtlich der Offenbarung und vor allem der Wunder, ohne deren bestätigende Funktion Gottes Reden im Munde Jesu nicht als solches angenommen werden kann - Fundamentaltheologie III/1, Münster 1981, 382; ähnlich sieht L. Scheffczyk keine Möglichkeit, das Geschichtshandeln Gottes unabhängig vom Glauben (das hieße ja auch, 
und heilend in die Menschheitsgeschichte eingreifen. Wenn denn der Begriff der Offenbarung angemessen ist für die Kennzeichnung des von Gott selbst initiierten Verhältnisses zur menschlichen Geschichte, dann impliziert dieser Begriff die prinzipielle Verständlichkeit der Botschaft. Sein sich Verständlich-machen-wollen und -können ist selbst Inhalt der Offenbarung. Damit wird die Tatsache der Offenbarung zu einem grundlegenden, alle weiteren konditionierenden Glaubensinhalt.

Wenn nun für die Annahme der Glaubensinhalte entscheidend ist, daß Gott sie mitgeteilt hat, sie dadurch ihren Sinn und ihre Heilsnotwendigkeit empfangen - und nicht dadurch, daß sie dem Verstand unnachvollziehbar sind -, dann liegt der Gedanke nahe, daß Gott nicht etwas oder alles über sich offenbart, sondern daß er sich in der liebenden Hinwendung zu den Menschen selbst offenbart. Er sagt nicht etwas, sondern bewirkt in seinem Offenbarungshandeln das Heil, die Erlösung der Menschheit.

Der entscheidende Grund für diesen Begriff der Selbstoffenbarung besteht darin, daß jede Unterscheidung, die die Theologie in Bezug auf Gott macht, nicht reale Verschiedenheiten in Gott aussagen kann, sondern nur verschiedene Aspekte seiner unüberbietbaren Seinsfülle approximativ erreichen kann. Jede Unterscheidung ist also nur analog, hat mehr von den Gesetzmäßigkeiten der menschlichen Vernunft in sich als daß sie die Wirklichkeit Gottes wiederspiegelte. Insofern kann zwischen der Art und Weise, wie Gott etwas mitteilt, und seinem innersten Leben keine grundsätzliche Differenz bestehen. Er offenbart nicht etwas (oder auch alles) über sich, sondern er vollzieht sich als das, was er ist, als absolute Liebe. ${ }^{14}$ Zwischen Tätigkeit und Sein kann in Bezug auf Gott nicht unterschieden werden, das wäre ein bloßer Anthropomorphismus.

Weil Gott adäquater ausgesagt wird, wenn er im dauernden Vollzug seiner Liebe begriffen wird, und wenn genau darin seine Unermeßlichkeit und Unendlichkeit für die menschliche Vernunft am ehesten bewahrt ist, dann ist auch in dieser Hinwendung Gottes zu den Menschen seine Einzigkeit und Absolutheit gegenwärtig. Das Heilser-

unabhängig vom Glaubenszeugnis der Jünger) positiv und endgültig beweisen zu können, so daß es als natürliches Motiv für einen übernatürlichen Glauben fungieren könne. Jedoch kann von dieser Nichtbeweisbarkeit keineswegs auf die Nichtwirklichkeit des Eingreifens Gottes in die Geschichte geschlossen werden - Die Theologie und die Wissenschaften, Aschaffenburg 1979, 115; zum Gesamtkomplex des Verhältnisses von Theologie und historischer Vernunft siehe G. Essen, Historische Vernunft und Auferweckung Jesu. Theologie und Historik im Streit um den Begriff geschichtlicher Wirklichkeit, Mainz 1995.

14 Siehe dazu $\mathrm{H}$. Peukert, Kommunikative Freiheit und absolute befreiende Freiheit, in: Wagnis Theologie. Erfahrungen mit der Theologie Karl Rahners (hg.v. H. Vorgrimler) Freiburg/Basel/Wien 1979, 277. 
eignis seiner Offenbarung ist also unüberholbar und endgültig - es kann sich nichts Größeres und Wichtigeres mehr in der Geschichte ereignen, was dieses Ereignis quantitativ oder qualitativ überböte, d.h. Selbstoffenbarung in diesem exponierten Sinne impliziert seine eigene Universalität in extensiver und intensiver Hinsicht.

Insofern ist der Offenbarungsbegriff nicht nur zu einem Gottesbegriff geworden, der Gott ganz meint, weil er seine verströmende Liebe als den inneren und äußeren Vollzug seiner Wirklichkeit benennt, sondern er ist zugleich Reflexionsbegriff. ${ }^{15}$ Jedes Reden über Gott weiß sich unter der Bedingung der Vorgabe göttlicher Rede. Nur dasjenige Sprechen erreicht seinen "Gegenstand", seinen Inhalt, das dessen Subjektivität und geschichtliche Wirksamkeit als den eigenen Ermöglichungsgrund realisiert.

\subsection{Wort Gottes}

In der gleichen Weise kann nun gefragt werden, was näherhin unter "Wort" verstanden werden muß, wenn Gott es spricht. Denn es gibt einen gravierenden Unterschied zwischen dem menschlichen und dem göttlichen Wort, der nicht juristisch oder durch Vollmacht überwunden werden kann, der auch dann bestehen bleibt, wenn Gott selbst in einer direkten Beauftragung einen Menschen sprechen läßt: Das menschliche Wort ist prinzipiell von der Wirklichkeit seines Inhaltes getrennt. Das göttliche Wort ist seine Wirklichkeit und umgekehrt hat Gottes Wirklichkeit ihr Erkanntsein, ihre symbolischen und intelligiblen Vermittlungen so in sich, daß Sein und Erkennen nicht unterschieden werden können.

Für Rahner ist das "Wort Gottes (als inneres Moment an der Heilstat Gottes am Menschen und so mit ihr und ihretwegen) ... das heilskräftige Wort, das an sich mitbringt, was es aussagt, ist selbst also Heilsereignis, das (in seinem äußeren, geschichtlichen und sozialen Moment) anzeigt, was in ihm und unter ihm geschieht und geschehen läßt, was es anzeigt. Es ist Gegenwärtigung der Gnade Gottes." 16 Es ist nicht satzhafte Belehrung, dessen "Sachverhalt... in seinem Bestand und seiner Gegebenheit von diesem lehrhaften Hinweis völlig unabhängig ist", sondern es ist "exhibitiv gegenwärtigendes Wort, in dem und unter dem die bezeichnete Sache allererst gegenwärtig ist." ${ }^{17}$

„Das Wort Gottes (auch im Munde der Kirche und ihrer Verkündigung) ist nicht zuerst und zuletzt Didache, sondern Proklama-

15 Siehe P. Eicher, Offenbarung. Prinzip neuzeitlicher Theologie, München 1977, 43ff; Kasper, Offenbarung 53 (Anm. 10).

16 K. Rahner, Wort und Eucharistie, in: ders., Schriften zur Theologie IV, Einsiedeln/Zürich/Köln 1960, 313-355, 321.

Ebd., 322. 
tion, in der die Ankunft des Proklamierten selbst geschieht, ist das machtvolle schöpferische Dabar Gottes an den Menschen, die Weise, in der sich die verkündigte Wirklichkeit selbst enthüllt und dadurch und so für uns anwesend wird, indem sie aus ihrer göttlichen Verborgenheit selbst heraustritt und so allererst da ist, wo wir sind." 18 Damit versteht Rahner das Wort Gottes in seiner absoluten Einheit mit der Wirklichkeit seines Inhaltes als Begriff der im neuen Testament berichteten Verkündigung Jesu. „Gegenüber jeglichem prophetischen Verkünden richtet der vom Vater gesandte Sohn (vgl. Lk 4,18.43) rechtsgültig und definitiv das in Gnaden vorausgesagte Heil auf: ,heute' (Lk 4,21) erfüllt sich die Befreiung der Gefangenen, die Heilung der Blinden, ausgerufen wird das ,Gnadenjahr des Herrn' (vgl. Is 61,1)."19

Wenn das Wort Gottes dermaßen berichtet wird und die Verkündigung Jesu, in der das Verkündete gegenwärtig wirklich ist, Norm für die Verkündigungspraxis der Kirche ist, dann ist es kein Grenzbegriff der auf Sinnlichkeit bezogenen Verstandestätigkeit, sondern eine Erfahrung, die von sich aus zum Begriff drängt. Die Augenund Ohrenzeugen der Realität, der "Nähe ${ }^{20}$ des Reiches Gottes konnten diese Erfahrung nur in universalen Kategorien aussagen und sich bewußtmachen.

18 Ebd., 323; siehe dazu auch F. Kamphaus, Der Gegenstand der Verkündigung, in: Pastorale. Handreichung für den pastoralen Dienst. Verkündigung (hg. im Auftrag der Deutschen Bischofskonferenz und der Konferenz der deutschsprachigen Pastoraltheologen) Mainz 1970, 60f (im folgenden: Pastorale etc.).

19 Lehmann/Rahner, Kerygma (Anm. 9) 624; auch ebd., 628.

Eigentlich müßte hier der Zusammenhang von der Verkündigung und dem Weg Jesu näher behandelt werden. Verkürzend kann gesagt werden: Die Proklamation des Gottesreiches als gekommen, als jetzt wirklich, erfährt seinen entscheidenden Gehalt und Grund in der "Sinneinheit von Tod und Auferstehung Jesu", d.h. in der Überwindung des Todes. Weil dies in der Auferstehung geschehen ist und als historische Realität angenommen werden kann (vgl. dazu die umfangreichen Reflexionen und Differenzierungen von Essen, Vernunft (Anm.13)), gibt es die Identität von Proklamation und der Gegenwart des proklamierten Reiches. Insofern ist Pröpper zuzustimmen, wenn er formuliert: Erst durch Jesu „Auferweckung vom Tod, die als Zeichenhandlung Gottes selbst gelten darf und unsere Vorstellung von ihm in realer Weise bestimmt, ist das Versprechen der Liebe, aus der Jesus lebte, für die er einstand und die er als Liebe Gottes identifizierte, in der denkbar äußersten Situation eingelöst worden. Erst durch sie hat sich Gott als die schlechthin unbedingte und schöpferische Liebe, als die ursprüngiche Liebe selbst für uns erwiesen." Th. Pröpper, Erlösungsglaube und Freiheitsgeschichte. Eine Skizze zur Soteriologie, München 1988, 198.

20 Siehe H. Merklein, Jesus, Künder des Reiches Gottes, in: Handbuch der Fundamentaltheologie II (hg.v. W. Kern, H. J. Pottmeyer, M. Seckler) Freiburg 1985, 145-174, 152f -im folgenden: HbFth + Band + Seite. 


\section{Glaube als Ziel der Verkündigung}

\subsection{Glaube als Wirksamkeit des Gotteswortes}

Das radikal verstandene Wort Gottes kann in einer Verkündigungspraxis, an der Menschen konstitutiv beteiligt sind (sei es als Subjekte, sei es als Hörer), immer nur von diesen Menschen in innerer Abwandlung und auf defiziente Weise "verwirklicht", nachgesprochen werden. Die Abgrenzung gegen seine Auffassung als satzhafte Wahrheit macht jedoch deutlich, daß es in dieser Defizienz Unterschiede gibt: Wenn die Trennung von Wirklichkeit und Wort dergestalt in Kauf genommen wird, daß sich das Wort gegenüber der Sache verselbständigt und unabhängig von ihr geglaubt und angenommen werden kann, und umgekehrt die Sache unabhängig von dem Wort spekulativ erschließbar ist, dann handelt es sich nicht mehr um Defizienz, sondern um einen Bruch. Die Trennung von Wort und Wirklichkeit unter den Bedingungen menschlichen Auffassungs- und Sprechvermögens muß also darin an der Einheit von Wort und Wirklichkeit festhalten, daß die Wirklichkeit nur über das Wort erreichbar ist, das ihr selbst entstammt. Und umgekehrt ist jedes Wort dann, wenn es der Einheit entstammt, nicht so von seiner Wirklichkeit gelöst, daß es nicht etwas von ihr erfahrbar machte.

Genau dieser Zusammenhang ist nach Rahner in dem scholastischen Topos der "Wirksamkeit des Wortes“ bewahrt: Diese Wirksamkeit ist zunächst darin zu suchen, daß das Wort selbst im Menschen seine Annahme bewirkt. „Mindestens die im Glauben gehörte Botschaft also ist für jeden Dogmatiker nicht bloß das Hören eines Satzes über etwas, sondern der Empfang der Wirklichkeit selbst, über die ein Satz gehört wird, und die bewirkt, daß ihre Mitteilung gehört und liebend geglaubt wird." 21 Dieser Empfang der Wirklichkeit des Glaubens kann nun einmal so gedeutet werden, daß damit die Defizienz menschlichen Daseins für das Nachsprechen des Gotteswortes grundsätzlich überwunden ist (und nur noch vom konkreten einzelnen Menschen selbst verspielt werden kann, nicht aber aufgrund seiner Natur ihm verschlossen ist), es kann aber auch zurückhaltender gedeutet werden als die Ermöglichung der Einsicht, daß im menschlichen Nachsprechen des Gotteswortes dessen Inhalt fehlt - also in der Befähigung des Menschen, seine Endlichkeit und Begrenzheit einzusehen.

Dennoch bliebe eine so verstandene Wirksamkeit des Wortes eigentümlich zirkulär, wenn sie lediglich ein (Glaubens-)Bewußtsein der Nichtwirklichkeit des Wortes in der Annahme durch den Menschen hervorbrächte. Will man nun die zu starke Deutung der Wirksamkeit als entitative Erhöhung des Menschen durch die Glaubensgnade vermeiden, dann bietet sich an, sie doppelt dimensioniert zu denken. Wirksam

21 Rahner, Wort (Anm. 16), 324. 
ist das Wort dann immer auf zwei Erfahrungsebenen, die "für uns" (die wir keine Augen- und Ohrenzeugen des Heilsereignisses sind) beide irreduzibel sind:

- Im Modus der Erzählung der Wirklichkeit, des Anbruches des Reiches Gottes in einem vergangenen Jetzt ist die Wirklichkeit im Glauben gegeben und zwar als im Glauben äußerliche Wirklichkeit. An die Erfüllung der Verheißung in Jesus Christus zu glauben heißt, $\mathrm{daB}$ die Wirklichkeit des Wortes Gottes geschichtlich erfahren worden ist. Das menschliche Nachsprechen des Wortes bezieht sich also auf eine punktuelle vergangene geschichtliche Wirklichkeit. In diesem Sinne ist es "teilweise“ mit Wirklichkeit gesättigt. Wirklichkeit besitzt das menschliche Wort also in einem vergangenen Ereignis, verschieden und getrennt von dieser Wirklichkeit bleibt es wegen des zeitlichen Abstandes. 22

- Wenn Rahner die Wirksamkeit des Gotteswortes darin sieht, daß es gehört und liebend geglaubt wird, dann ist das ein entscheidender Hinweis auf dessen innere Wirklichkeit. Die innere Wirksamkeit des Gotteswortes ist dann nicht primär das Wissen um seine Unverfügbarkeit und Unableitbarkeit aus dem menschlichen Willen und Bewußtsein, auch nicht eine bloß verborgene Seelenqualität, die für niemanden einsehbar ist als für den, der sie hat, nicht eine bloße Potenzialität, sondern lebendiger Impuls zu einer Praxis der Gottesund Nächstenliebe. Die innere Wirksamkeit des Wortes Gottes im Glauben besteht darin, daß dieser durch die Liebe geformt ist. Wenn Liebe und Glaubensbewußtsein zusammentreten wie Form und Materie, dann ist eine Realitätsdichte erreicht, die in Kontinuität zu der steht, die im Gotteswort angenommen wird. ${ }^{23}$

Der Unterschied zwischen dem, was für die Jünger an Heilsfülle in der Mitte der Zeit gegenwärtig war und was in der Praxis des Glaubens wirklich wird, ist also kein modaler (es sind also nicht verschiedene Seinsmodi wie Wirklichkeit und Möglichkeit), sondern es sind Unterschiede in der Wirklichkeitsdichte, in der Intensität und Extensität von

22 lm der Annahme des Wortes als Hören auf die Erzählung verwandelt sich die in der Form des Wortes mitgeteilte Nichtwirklichkeit in das Vergangensein des Inhaltes.

23 Siehe dazu P. Knauer, Der Glaube kommt vom Hören. Ökumenische Fundamentaltheologie, Freiburg 1991, 165ff. Diese Realitätsdichte ist zunächst in der Liebe des Menschen zu Gott erreicht: Weil Gott den Menschen liebt, realisiert er diese Liebe für sich nur dann adäquat, wenn er ihr zu entsprechen sucht, also seinerseits Gott liebt. Weil er aber einer absoluten und universalen Liebe zu entsprechen sucht, die ja nicht nur ihm als einzelnem gilt, sondern auch allen anderen menschlichen Individuen, deswegen impliziert die Gottesliebe notwendigerweise die Nächstenliebe; siehe dazu B. Schüller, Die Begründung sittlicher Urteile, Düsseldorf 1973, 28. 
Wirklichkeit. Für den, der das Heil erfährt (im Akt der Nächstenliebe aus dem Glauben oder in der Unmittelbarkeit zum Offenbarungsträger) ist das Wort Gottes es selbst - wenn vielleicht auch nur einen Augenblick im präzisen Sinne einer Zeitbestimmung.

\subsection{Praxis gegen die Ursachen von Leidenserfahrungen}

In der universalen Erfahrung des Heils (vorstellbar nicht unter der Bedingung von Zeit, sondern vielleicht nur im Augenblick ihrer Stillstellung, Unterbrechung, im Augenblick des messianischen Eingreifens in die Geschichte) exponiert sich das menschliche Subjekt auf extreme Weise der Widerlegung dieser Erfahrung. Jedes Unrecht, das auf der Welt existiert, und jedes Leiden, das je erfahren wurde, hat dann prinzipiell den Status eines Gegenarguments. Es ist eine Einschränkung der Universalität der Heilserfahrung.

Das Gegenteil dieser Erfahrung des Heils ist ein Verhalten, daß seine theoretische Möglichkeit denkt und praktisch alles tut, um seiner Gefährdung durch widersprechende Realitäten auszuweichen. Das ist leicht als intellektuelle Selbstbevollmächtigung des Menschen zur universalen Heilerfahrung deutbar und einsehbar. Der Verzicht auf Anschauungen und Erfahrungen ist kein Gnadengeschenk, sondern ein menschlich hybrider Versuch, die Universalität des Heils herbeiführen zu wollen.

Normalerweise wird diese Hybridität jedoch an einer Praxis festgemacht, mit der alle Leidensursachen beseitigt werden sollen und mit der das Bewußtsein einhergeht, daß dieses auch menschenmöglich sei. In einer historischen Gestalt dieser Praxis, in bestimmten Strömungen des Marxismus, sollte nun diese Möglichkeit dadurch zur realen Fähigkeit der menschlichen Gattung avancieren, daß faktische Leidenserfahrungen geleugnet, wegerklärt oder methodisch gezielt ausgeblendet wurden.24 Damit scheint jeder menschliche Versuch, in einer geschichtswirksamen Praxis Leiden beseitigen zu wollen, desavouiert. Weil im historischen Projekt des Sozialismus der säkulare Wille eines Klassensubjektes sich durch die Leugnung von Leidenserfahrungen selbst behauptete und konstituierte, letztlich sich täuschte, deswegen ist aber die praktisch werdende Sehnsucht und der Protest gegen leidverursachende Zustände noch nicht unvereinbar mit der kirchlichen Verkündigung. Vielmehr hat sie einen hohen Stellenwert und ist genau dann mit ihr verknüpft, wenn in dieser Praxis die Möglichkeit des Scheiterns in Rechnung gestellt wird. Das gilt umso mehr für eine Praxis, die die Sensibilität für reale Leiden steigert und durch die Leidenssituationen aufgespürt und angeklagt werden.

24 Diese Versuche dokumentieren große Teile des Werkes von Alexander Solschenizyn. 
Diese Praxis hat nur dann, wenn sie wirklich stattfindet und angesichts ihres Scheiterns, angesichts ihrer ebenso realen Unzulänglichkeit durchgehalten wird, einen Zusammenhang mit der im Evangelium bezeugten Erfahrung des universalen Heils. Sie ist mit dieser Erfahrung modal identisch - wie oben festgestellt -, nämlich wirklich. Und diese Modalität, wirklich stattzufinden, gestattet es, daß sie Objekt empirischer Erkenntnis ist. Sie muß beschrieben und erfahren werden können. Es muß möglich sein, auf ihre raum-zeitliche Bestimmtheit hinzuweisen. Als solche unterscheidet sie sich von einer bloß projektierten, nur gewollten oder gesollten Praxis, die einzig darin sich vollzieht, den Willen anderer zu setzen und sie von dem Sinn dieses Sollens zu überzeugen. Sie ist nicht nur bewußtseinsverändernd, sie führt nicht nur zu einer neuen Weltsicht.

Dennoch ist diese wirklich vollzogene Praxis nicht identisch mit der Heilserfahrung. Der modale Zusammenhang gestattet es nicht, nur Entsprechungen zu sehen. Er ist zugleich die Basis dafür, ihren je unterschiedlichen Intensitäts- und Extensitätsgrad zu ermessen. Die Erfahrung des Heils war eine Erfahrung des Heilwerdens der ganzen Wirklichkeit, eine Erfahrung der Neuschöpfung des Kosmos in einem Augenblick der Menschheitsgeschichte. Von diesem Augenblick bewegt sich die Geschichte weg. Jede Zeit, die vergeht, vergrößert den Abstand zu dieser Wirklichkeit. Die in der Verkündigung bewirkte Praxis ist gerade in ihrer Gegenwärtigkeit wirklich, jedoch nur in der Lage, vorübergehend und unvollständig die gefallene und leidende Natur wiederherzustellen.

Auf der Basis des modalen Zusammenhanges bleibt die Glaubenspraxis von der Heilserfahrung verschieden und auf sie verwiesen. Wie dieser Verweisungszusammenhang näherhin aussieht, soll im folgenden in zwei Schritten reflektiert werden: Im 4. Abschnitt wird gefragt, wie die Wirksamkeit des Wortes näherhin zu begreifen ist und welche vermittelnde Rolle dabei die Botschaft von der vergangen universalen Heilserfahrung spielt, im 5 . Abschnitt, welche Bedeutung diese Praxis dafür hat, daß die Erzählung dieser vergangenen Erfahrung als wahr erkannt werden kann und ihre Universalität auch "für mich" gilt.

\section{Die Bedeutung der Erzählung}

\subsection{Verkündigung und Freiheit}

Für die Verkündigungstheologie der dreißiger und vierziger Jahre im katholischen Raum ${ }^{25}$ ist der konkrete Verkündigungsakt dann authen-

25 Einen Überblick unnd zugleich eine Kritik auf der Basis der Neuscholastik siehe bei E. Kappler, Die Verkündigungstheologie. Gotteswort auf Lehrstuhl und Kanzel, Freiburg 1949. 
tisch, wenn er Gott als erstrebenswertes Ziel "aussagt“. Und Gott als Ziel, als summum bonum auszusagen oder im Glauben anzunehmen heißt, es im Handeln anzustreben.

Wenn nun dieses bonum nicht im Rückschluß von der Schöpfung auf deren Urheber (im Rückschluß von der "besten aller Welten ", als die Gott die Welt nur schaffen konnte, auf ihren Ursprung) angezielt wird, sondern mit ihm Gott selbst gemeint ist, dann ist dieses Handeln frei. Freiheit heißt hier zunächst, daß die Mittelwahl nicht mehr äußerlich determiniert ist. Das Ziel, das angestrebt ist, gibt nicht mehr vor, wie gehandelt werden muß.

Diese Freisetzung kann man auf zweierlei Weise denken: 1. Wenn Gott als absolute Liebe menschliches Handeln determiniert, es als Handeln zu sich als dessen Ziel bestimmt, dann teilt er dem Handlungssubjekt zugleich seine Souveränität und Kompetenz mit, Ziele zu setzen und Mittel zu ihrer Erreichung zu wählen. Von Gott bestimmt $\mathrm{zu}$ werden heißt, in die Lage versetzt $\mathrm{zu}$ werden, auch das höchste Ziel mit „eigenen“ Mitteln erreichen zu können.26 2. Oder die menschlichen Mittel reichen prinzipiell nicht mehr aus, Gott zu erreichen. Der Mensch ist spätestens dann, wenn er dies von sich aus will, auf sich selbst zurückgeworfen. Keine objektive Ordnung garantiert ihm die Angemessenheit seiner Wahl. Darin erwächst ihm das Bewußtsein, für die Handlungen und Ziele selbst zuständig zu sein. Umgekehrt weiß er, daß Gott sich selbst ihm zuwenden muß, sich selbst als Mittel zur Erreichung des Ziels, das er selbst ist, anbieten muß, wenn sein Handeln auf Gott hin irgend einen Sinn und einen wie bescheidenen Erfolg auch immer haben soll. In der Begegnung mit diesem absoluten Mittel bzw. der Selbstvermittlung Gottes in der Geschichte ist dann menschliches Streben nach Gott am Ziel.

Was ist das für ein Handeln, das sich einerseits unendlich vollziehen muß, um ins Ziel gelangen zu können (wobei dieses Ziel selbst noch einmal nicht Ordnung, sondern ein Selbstvollzug, eine Bewegung ohne jede Potenzialität ist), anderseits aber den sich selbst geschichtlich zur Verfügung stellenden Gott der absoluten Liebe als Mittel „zur Verfügung" hat. Wenn die unendliche Verschiedenheit von Gott und Mensch nicht in der Begegung mit der sich selbst mitteilenden Liebe in eine Gottesunmittelbarkeit aufgehoben sein soll (wie in der visio beatifica), dann muß innerhalb des die göttliche Liebe anstrebenden Handelns unterschieden werden. Es muß zwischen einem Handeln, das bloß gesollt und gewollt ist (das sich bloß als Denkmöglichkeit zur Wirklichkeit drängt, ohne daß das Denken für seine Wirklichkeit

26 Gott wäre so die Formalursache für das menschliche Verlangen nach Gott siehe dazu K. Rahners Theorie des übernatürlichen Existenzials in seinem Grundkurs des Glaubens, Freiburg 1976, $132 \mathrm{ff}$. 
einstehen könnte) und einem wirklichen Handeln differenziert werden. In der Verwirklichung ist der Inhalt der Möglichkeit nicht vernichtet, wohl von dem Mangel, bloß Möglichkeit zu sein, befreit. Anderseits kommt dieser Möglichkeit ihre eigene Wirklichkeit nicht selbst zu, sondern bleibt geschenkt, muß - letztlich - durch ein absolutes Subjekt vermittelt sein.

Von daher'kann gesagt werden, daß die Annahme des summum bonum im Glauben nur dann erfolgt, wenn das Glaubenssubjekt ihn tatsächlich vollzieht. Im Glaubensakt bleibt es jedoch frei; der Glaube ist sein Akt, den es aufgrund seiner natürlichen Ausstattung wollen kann, dessen Vollzug das menschliche Subjekt dem göttlichen Gnadenhandeln verdankt. Gott habe ich also noch nicht gehört, wenn ich lediglich die Möglichkeit in mir erkenne, von ihm angesprochen zu werden und auf seine Ansprache frei antworten zu können; sondern nur dann höre ich ihn, wenn ich mich als tatsächlich angesprochen erfahre und im identischen Akt mich dementsprechend mir und meinem Nächsten gegenüber verhalte. Solange ich Verhaltensmöglichkeiten erwäge, habe ich den Anruf noch nicht wirklich vernommen.

Eine derartige Spezifizierung der Heilswirksamkeit der Predigt scheint in dezisionistischer Unmittelbarkeit zu münden: der Spielraum bzw. die Distanz, die Freiheitsbewußtsein immer bedeutet, scheint übersprungen - der Freiheitsvollzug dann bewußtlos, und in dem Sinn wohl von mir faktisch vollzogen, nicht aber verantwortet und frei. Denn es gibt keine Freiheit ohne Freiheitsbewußtsein.

Gelöst werden kann dieses Problem, wenn in Rechnung gestellt wird, daß in der heilswirksamen Verkündigung immer der Einzelne angesprochen wird, aber nicht als einzelner, sondern in seinen geschichtlichen und gesellschaftlichen Abhängigkeiten und Bezügen. Die Heilswirksamkeit gilt also nicht allein für mich, sondern immer auch für viele andere einzelne, die untereinander verbunden sind. Wirksam ist die Predigt nicht erst dann, wenn sie von allen Individuen in konkreter Praxis realisiert wird, sondern schon, wenn wenigstens einige, vielleicht wenige dies getan haben.

Das heißt, heilswirksame Predigt richtet sich dadurch an die Freiheit des Einzelnen, daß sie von der Bestimmtheit dieser Freiheit in der Praxis anderer einzelner erzählt. Nur darin ist das Streben nach Gott nicht bloß Projekt, das ich für mich erwäge (allerdings immer auch erwägen und verantworten muß), sondern das andere vollziehen oder vollzogen haben.

Und genau dieses geschichtlich reale Streben nach Gott als dem letzten Ziel des menschlichen Lebens überhaupt ist dann das inhaltliche Moment der Heilspredigt. Nur eine wirkliche Praxis der Nächsten- und Gottesliebe kann die Freiheit des Menschen so ansprechen, daß sie anerkannt wird und ihr Gott als höchstes Gut, als ihre eigene 
Möglichkeit real wird - schärfer formuliert: daß ihre eigene Möglichkeit bereits geschichtliche Realität ist. ${ }^{27}$

\subsection{Die Wirksamkeit der Erzählung}

Damit entsteht die Frage, wie die Erzählung in der Verkündigung Menschen anspricht und heilswirksam ist.

Auf den ersten Blick ist von der Wirksamkeit der Erzählung in der Erzählung selbst die Rede: von der Bekehrung und der Praxis der Jünger - wobei freilich nicht die Erzählung in ihrer literarischen und redaktionellen Endgestalt vor ihrer Entstehung bereits in ihren Inhalten selbst wirksam wäre, sondern die Redaktion der Evangelisten dokumentiert die Wirkung der Protoerzählungen, des ursprünglichen Gotteswortes. Insofern hat die Erzählung selbst eine transzendental-reflexive Struktur. Die Endredaktion führt sich selbst auf die Wirkung des redigierten Stoffes zurück. Die Verschriftlichung der mündlichen Tradition ist eine Wirkung der mündlichen Tradition - das, was in jenen Tagen in Jerusalem geschah, mußte aufgeschrieben werden.

Als tradierte Erzählung eines vergangenen Geschehens drängt die konkrete Verkündigungspredigt von außen an den Hörer heran. Sie ist neu und als solche Anrede an Menschen, die diesen Inhalt nicht kennen und nicht realisiert haben. ${ }^{28}$ Dabei versichert die Erzählung dem Hörer,

27 Mit der Annahme, daß der Mensch im Glauben Gottes Heilswirklichkeit entsprechen muß (und nicht einem von Gott an ihn gerichteten Imperativ), scheint zumindest die Wahlfreiheit verloren: Zwischen Heil und Verdammnis kann man nicht wählen wie zwischen innerweltlichen dinglichen Objekten oder konkurrierenden Handlungszielen. Formal unbedingte Freiheit und Autonomie wird davon nicht relativiert oder in Heteronomie aufgelöst - das wird nur dann zum Problem, wenn sie durch den wirklichen Vollzug der Freiheit in einem anderen Subjekt erzeugt würde. Der unbedingten Freiheit "leuchtet" nach Pröpper eine Glaubenspraxis unmittelbar ein, in der Gottes ergangenes Wort und geschichtswirksames Heil einzig angemessen zur Darstellung kommt. Als Darstellung göttlicher Liebe ist sie der Vollzug jener Freiheit, die der Mensch als seine ureigenste Möglichkeit denken, deren Realität er aber selbst nicht gewährleisten kann - siehe dazu Pröpper, Erlösungsglaube (Anm. 19) 194.

28 Genau dieser Dimension des verkündigten Textes selbst muß immer wieder in der Verkündigungspraxis betont und vollzogen werden: nur in der Neuheit des Textes erfährt der Leser, daß dieser von außen kommt, daß Gott etwas mit ihm vorhat und nicht einfach nur das bestätigt, was vorhanden ist. Der als neu empfundene Text kann die Erwartung wecken, daß im Verstehens- und Auslegungsproze $B$ des Textes mit mir als Leser und Ausleger real etwas geschieht.

Für Bastian ist diese Neuheit des Textes so essentiell und für die Verkündigungspraxis konstitutiv, daß seine Wahrnehmung als Bestätigung von Bekanntem ein Mißverständnis wäre. Es gehört deshalb zu den basalen Aufgaben der Verkündigung, diese Neuheit der biblischen Botschaft zu 
daß er diese Neuheit und Fremdheit auch für sich realisieren kann. Denn genau dieses Ankommen des Gotteswortes beim Menschen ist ihr zentraler Inhalt, ihre inhaltliche Struktur. Sie erzählt Gottes Liebe als Wirkung in der Umkehr der Jünger. In der Umkehr und der Veränderung des eigenen Lebens wird die Fremdheit der Erzählung bewahrt und zugleich meine Realität, in meiner Umkehr- und Veränderungspraxis real.

Wie wirkt nun die Erzählung auf die gegenwärtigen Hörer und welche Freiheitsspielräume haben sie, ohne daß die Wirkung bloß neutrale Vorlage ist, gegenüber der man sich so oder so verhalten kann? Um eine Antwort zu skizzieren, muß zuvor die Frage noch schärfer gefaßt werden. Sie zielt nicht darauf, wie ein Verkündigungssubjekt mit dem Inhalt Wirkungen erzielen kann. Es ist nicht nur eine Frage nach dem richtigen Umgang mit dem Text, damit er erfolgreich ist, sondern es ist die Frage nach der Wirkung des Textes selbst. Denn sonst wäre die Wirksamkeit des Wortes nur teilweise in den Inhalt der Erzählung eingegangen, nur als Bericht, als Protokoll, als Information. ${ }^{29}$ Der Einsatz und die Verwendung des Textes erforderte nochmal ein eigenes,

sichern. Sie erreicht dieses, indem sie den Text verfremdet - im Anschluß und in Kontinutät zu biblischen Verfremdungseffekten, zu einer in der Erzählung des Heilsereignises bereits dokumentierten Bewahrung der Neuheit des ewigen Wortes für den Menschen - siehe dazu H. D. Bastian, Verfremdung in der Verkündigung, in: HbV I 97ff.

29 Insofern ist Skepsis geboten gegenüber den Verkündigungstheorien, die den Inhalt selbst als Information und die Art und Weise, in der er übermittelt wird, als Informieren erachten. Das ist eine Modernisierung eines neuscholastischen Inhaltsverständnisses. Den Inhalt braucht man nur zur Kenntnis zu nehmen. Durch dessen Zurkenntnisnahme ändert sich aber nichts, wenn die Befähigung zum Umgang mit dem Text nicht längst vorher durch einen eigenen Gnadenakt Gottes gewährleistet wäre.

Wenn Bastian meint, den Verfremdungeffekt in der Gegenwart damit sichern zu können, daß die Botschaft als Nachricht und Information an die Hörer weitergegeben wird, dann verkennt er den Distanzierungs- und Objektivierungsvorgang, der in der Verwandlung eines Ereignisses in eine Information stattfindet.

Dieser Distanzierungsvorgang kann mit Karl Kraus und Walter Benjamin beschrieben werden: Nach Benjamin gibt es - im Rückgriff auf Kraus - die sprachliche Form der Information erst in dem Augenblick, in dem es bestimmte Kommunikationstechniken und -mittel gibt. Die Presse ist für ihn die Bedingung dafür, daß über etwas informiert werden kann. Zugleich emanzipiert sich in der Information die Mitteilung von ihrem geschichtlichmateriellen Substrat und inszeniert sich selbst als Neuigkeit, als Sensation. Eine Neuigkeit, die sich vom Inhalt emanzipiert hat, wird dadurch mit anderen Neuigkeiten identisch und immergleich. Die Presse bzw. die technischen Massenmedien sind die ewige Wiederkehr des Neuen, die immergleiche Neuigkeit - siehe dazu W. Benjamin, Gesammelte Schriften II (hg.v. R. Tiedemann, H. Schweppenhäuser) Ffm 1977, 344f. Die Auffassung der Botschaft als Information scheint - wenn denn die Benjaminsche Analyse stimmt - das Anliegen der Verfremdung zu konterkarieren. 
nicht im Text enthaltenes Wissen. Hier handelt es sich also um die Frage nach der Identität von Inhalt und Wirkung, von erzähltem Geschehen und Geschehen selbst. Es ist letztlich die Frage nach der Einheit des Verkündigungsaktes.

Auf dieser Basis läßt sich nun erörtern, inwiefern die Verkündigung ein Sprechakt ist bzw. inwiefern die Sprechakttheorie ${ }^{30}$ eine angemessene Darstellung des Verkündigungsgeschehens und ihrer Wirkungen ermöglicht: Einerseits gibt es eine Berechtigung, die konkrete Verkündigungspraxis mit einer derartigen Theorie zu beschreiben: Die Entdeckung, daß mit Aussagen auch immer Wirkungen intendiert sind, trifft eine Dimension derjenigen Aussagen, die in der Verkündigung gemacht werden. ${ }^{31}$

Anderseits muß man unterscheiden zwischen intendierter und tatsächlicher Wirkung - für die Sprechakttheorie ist die Frage, ob ein Hörer einer Aussage auch wirklich die mit der Aussage intendierten Wirkungen vollzieht, dem Sprachgeschehen äußerlich. ${ }^{32}$ Nun ist das genau der springende Punkt, daß in den Verkündigungsinhalten gerade diese konkreten Wirkungen inhaltlich sind und so konkret gedacht werden müssen, daß sie erzählt werden können. Sie sind nicht nur in der Erzählung gemeint, sondern abgebildet und sprachlich wiedergespiegelt. Düsterfeld sieht die Mangelhaftigkeit der Sprechakttheorie darin, daß diese auf der Satzebene ausgebildet worden ist: Sie analysiert die Wirkungsintentionen von Sätzen wie "Der Hund ist bissig" etc. Und Urteile erzeugen Kompetenzen, Fähigkeiten. Sie sind die Bedingung der Möglichkeit, bestimmte Handlungen durchzuführen, nicht aber deren Durchführung selbst. ${ }^{33}$

Der Vorschlag Düsterfelds besteht nun darin, einerseits die Wirkungsintention, die die Sprechakttheorie in Urteilen festmacht, auf die Ebene des Textes, der Erzählung zu transponieren und anderseits die Frage zu stellen, wie sich Erzählungen von tatsächlich vollzogener solidarischer Praxis und deren Stattfinden in der Gegen wart zueinander verhalten. ${ }^{34}$

30 Siehe die ausführliche und präzise Darstellung bei E. Arens, Kommunikative Handlungen. Die paradigmatische Bedeutung der Gleichnisse Jesu für eine Handlungstheorie, Düsseldorf 1982, 173-322.

31 R. Zerfaß, Die Sprache der Verkündigung, in: Pastorale (Anm. 18) 81-96; J. Thomassen, Heilswirksamkeit der Verkündigung. Kritik und Neubegründung, Düsseldorf 1986; P. Düsterfeld, Predigt und Kompetenz. Hermeneutische und sprachtheoretische Überlegungen zur Fundierung einer homiletischen Methode, Düsseldorf 1978.

32 Thomassen, Heilswirksamkeit (Anm. 31) 230.

33 Düsterfeld, Predigt (Anm. 31) 136.

34 Ebd., 168; insofern muß streng unterschieden werden zwischen einer pragmatischen Verkündigungstheorie und der Anwendung der Pragmatik für die Auslegung der biblischen Sprechsituationen und - handlungen. Nach 
Wie kann die konkrete gegenwärtige solidarische Praxis in der Erzählung stattfinden, ohne daß sie zu einem bloß vergangenen Geschehen, das die Zeit in der Modalität der Sprache und der Erkenntnis überdauert, depotenziert wird? ${ }^{35}$ Als Erzählung sind die biblischen Texte zum einen gegenstandsorientiert, inhaltlich. Sie sind Erzählung von etwas. Zum anderen sind sie selbstimplikativ. Erzähler und Hörer sind in der Erzählung mitgemeint. Letzteres nicht in dem Sinne, daß das Erzählen immer auch (zusätzlich und möglicherweise) ein performativer Akt ist, sondern in dem Sinne, daß Zuhörer und Erzähler selbst Inhalt sind.

Wenn Verkündigung ein einheitlicher Akt ist und sich nicht aufspalten läßt in einen darstellenden und einen davon getrennten wirkenden, dann muß der Inhalt so beschaffen sein, daß es evidentermaßen falsch ist, ihn nur zur Kenntnis zu nehmen. ${ }^{36}$

Ein Beispiel dafür ist ein Text, der eine Gefahr berichtet, die mir, dem angeredeten Subjekt droht. Wenn ich dieses nur zur Kenntnis nehme und keine Konsequenzen zeitige, keine Schutzmaßnahmen gegen die Gefahr treffe, habe ich ihn falsch verstanden. Zu seinem Verständnis gehört also eine Praxis, mit der ich mich vor der Gefahr schütze. In Analogie dazu kann gesagt werden: Die biblischen Texte berichten von einer Praxis, die mir gilt. Weil ich in dem Text gemeint bin, kann ich ihn nicht objektivieren. Ich würde mich von außerhalb meiner selbst betrachten, wenn ich den Text meiner Rettung, der mir geltenden befreienden Praxis nur zur Kenntnis nähme (als etwas, das mich nichts angeht).

Das darf nun nicht als moralischer Appell, die Texte richtig zu verstehen, mißverstanden werden. Durch folgenden Gedankengang mag dies einsichtig werden: Wenn es nicht meine Interpretationsleistung ist, mich in der Erzählung wiederzufinden, dann muß mein Name in ihr vorkommen, dann bin ich nicht nur gemeint und vom Text angezielt, sondern mein Leben ist bereits als gelingendes, mein Heil ist

Arens können die Gleichnisse Jesu deswegen als innovatorische Sprachhandlungen beschrieben werden, weil sie die Basileia "abbilden" und die "Wirklichkeit Gottes, die von seiner mit Jesus angebrochenen Herrschaft nicht mehr getrennt werden kann", "erschließen". Sie "zeigen auf”, welches Handeln und Verhalten der gleichzeitigen "Erfahrung” der Herrschaft Gottes entspricht. Als solches sind sie also weder Appelle noch von der Wirklichkeit isolierbare Sprachereignisse, sondern ein Sprechen, das seine eigene Wahrheit aufdeckt und sichtbar macht, weil diese gegenwärtig ist siehe dazu Arens, Handlungen (Anm. 30) 351f.

$\mathrm{Zu}$ einer theologischen Erzähltheorie siehe J. B. Metz, Glaube in Geschichte und Gesellschaft. Studien zu einer praktischen Fundamentaltheologie, Mainz 1977 u.ö. 181-194.

36 „Eine Glaubensaussage dagegen nur hypothetisch verstehen zu wollen, heißt bereits, sie mißzuverstehen." Knauer, Glaube (Anm. 23), 361. 
bereits in ihm mitgeteilt. Das kann so aufgefaßt werden, daß ich mein Leben durch die Erzählung neu sehe. Die Wirkung einer solchen neuen Sicht meines Lebens als gelingend besteht dann darin, daß ich mich von nun an so verhalte, daß mein Leben tatsächlich gelingt. Dieses Verhalten unterscheidet sich vom zielgerichteten Handeln dadurch, daß es Mimesis einer für real gehaltenen Wirklichkeit ist. Die mitgeteilte Vorhersage des Gelingens meines Lebens ist die Ursache dafür, die dementsprechenden Kräfte zu mobilisieren. Dabei besteht die Gefahr, daß die Wahrheit dieser Erzählung nur dadurch unwiederlegbar und absolut bleibt, dảß jedes Mißlingen dieser Praxis meiner unvollständigen Annahme der Fiktion, dem unvollständigen Sich-berühren-lassen des Hörers angelastet wird.

Wenn die Erzählung darin universal ist, daß mein Name in ihr vorkommt und mein Leben als gelingend erzählt wird, gleichzeitig der Hörer dieses aber nur als Prospektion einer Zukunft auffassen kann, dann exponiert sich eine so gedachte Wirksamkeit der Botschaft der doppelten Rückfrage: Wo steht mein Name und was wird über mich erzählt? Allein die Tatsache, daß offensichtlich die menschliche Natur so beschaffen ist, daß sie das Gotteswort, in dem auch mein ganzes Leben als gelingend und gerettet ausgesagt ist, nur als Prospektion begreifen kann, gibt mir das Recht zu dieser Frage. Denn wer eine Prospektion auf ihre Adäquanz mit der Wirklichkeit befragt, ist immer im Recht und nicht erst der, der bereits auf ein Scheitern zurückblickt.

Diese Frage, zu der der Hörer deswegen berechtigt ist, weil er seine Rettung, sein Heil zunächst nur als Vorhersage in der Erzählung vom universalen Heil lesen kann, kann nun nicht selbst wieder die Antwort liefern. Es muß einen Anhaltspunkt in der Wirklichkeit geben, die jetzt schon da ist. Es muß eine Praxis geben, die der Rettung des Hörers gilt, in der der Hörer sich als geliebt erfährt. Genau darin wird die Vorhersage zur Verheißung gesteigert. Und nur als Verheißung ist die Erzählung inhaltlich universal. Denn nur wenn sie Momente der Erfüllung auch meines Glücksverlangens bereits enthält, kann ihr universales Ergangensein erzählt werden.

\section{Die Bedeutung des Zeugnisses für die Wirksamkeit und Wahrheit der Verkündigung}

\subsection{Die Unselbständigkeit der Erzählung}

Wenn ich in der Botschaft gemeint bin, dann muß das also - das erbrachten die Überlegungen von 4.2 - verständlich und nachvollziehbar sein. Ein Anhaltspunkt für die Verständlichkeit der Behauptung, $\mathrm{da} B$ das Wort Gottes meine Rettung aussagt (daß das Gelingen meines Lebens in der Objektivität der Erzählung vorkommt), ist eine Praxis, die mir gilt, die ich am eigenen Leibe erfahre. 
Diese Praxis ist nicht meine eigene. Nicht ich bin ihr Urheber, sondern ihr Empfänger. Es ist nicht der Appell an mich, dieses oder jenes zu tun, sondern sie ist bewirkter Glaube in einem anderen Subjekt, der sich rettend auf mich auswirkt. Es ist insofern für mich objektiv und mir äußerlich, vorgegeben.

Die Konkretheit dieser Praxis ist für mich nicht Aufgabe, sondern diese Praxis ist relevant, heilend und rettend für mich, weil sie konkret ist - und nur deswegen. Ihre Geltung für mich hat also ihren Grund in ihrer raumzeitlichen Bestimmtheit.

Diese Praxis ist also auch kein Projekt eines Anderen oder Anderer, sondern ein Handeln, das mich bereits erreicht hat und auf das ich deswegen zurückschauen kann. Der andere will mir nicht helfen, sondern er hilft mir. Diese Praxis ist in ihrem Wirklichsein symbolisch, nicht in ihrem Anspruch auf extensive Universalität.

Wenn die solidarische Praxis deswegen ein Anhaltspunkt der Wirklichkeit des Heils im Offenbarungswort ist, weil sie wirklich ist, dann kann man versucht sein auch umgekehrt zu schließen: Jedes Handeln, das in der materiellen und geistigen Einheit vollzogen wird, ist wegen dieser Einheit wirklich, und aufgrund dieser Modalität fungiert es als Anhaltpunkt für göttliches Offenbarungshandeln. Dieses Kriterium erfüllen aber auch Handlungen, deren materiale, akzidentelle Bestimmungen nicht mehr mit Eigenschaften Gottes zusammenhängen. ${ }^{37}$

Die Folge wäre, daß banale Akte der Nettigkeit wie Geschenke zum bürgerlichen Weihnachtsfest etc. schon die Wahrheit der Wirksamkeit des Gotteswortes verbürgen und bestätigen würden - sie brauchten lediglich wirklich zu sein und ihre Wirklichkeit müßte vom Betreffenden, vom Hörer bestätigt werden. Es taucht also die Frage auf, ob es Kriterien gibt, welche Praxis die Bedeutung eines Anhaltspunktes für die Wahrheit der Botschaft haben kann. Daß sie mir gilt, also auf einen einzelnen Menschen als ihr Ziel gerichtet ist, ist so verstanden noch ein leeres Kriterium. Denn diesem Kriterium genügt auch ein Handeln, daß das Leben bedroht und zu vernichten trachtet. ${ }^{38}$

37 Normalerweise wird die Kontinuität des verkündeten Wortes mit dem Gotteswort in materialer Hinsicht gesehen. Verschieden ist es dann in seiner Form; die Verkündigung und Erzählung des göttlichen Erlösungshandelns wird dann immer in modo cognoscendi ausgesagt - s.o. Wenn nun die Kontinuität zum Gotteswort darin festgemacht wird, daß das menschliche Wort nicht Wort, sondern Praxis ist, an der Geist und Leib, Verstand, Wille und vorfindliche Welt ganzheitlich beteiligt sind, dann scheint dieses nur auf Kosten der materialen Kontinuität denkbar.

38 Causa finalis und causa materialis sind nicht auf einer Ebene auftragbar. Die materiale Bestimmung der Praxis bestünde darin, daß sie irgendwie mit den Eigenschaften Gottes zusammenhängt - darunter fällt die Finalität genau nicht, stellt also ein eigenes Konstitutionselement dar. 
$\mathrm{Zu}$ diesem Einwand ließe sich sehr viel sagen - die entscheidende Entgegnung lautet: Die wirkliche Praxis ist nicht aus sich Verweis auf die universale Heilswirksamkeit des Gotteswortes, sondern nach ihr wird gefragt, weil zuvor die gehörte sprachlich vermittelte Botschaft bezweifelt wurde. Ihre extrem zugespitzte Behauptung, daß mein Leben in ihr als gerettet erzählt wird, berechtigt den Hörer dazu, danach zu fragen, ob es stimmt. Und dabei ist die wirklich mir geltende Praxis der Nächstenliebe der einzig mögliche benennbare Anhaltpunkt. Die Frage nach innerweltlicher, geschichtlicher Identität von Form und Inhalt entstammt also der Frage, daß die göttliche Materie, die Eigenschaften Gottes, die inhaltliche Kontinuität der Verkündigung nur nach menschlicher Art, sprachlich gegeben sein kann. ${ }^{39}$ Der garstig breite Graben der unaufhörlich vergehenden Zeit trennt die Glaubenden von der Wirklichkeit ihres Glaubensinhaltes.

Diese Praxis ist also nicht an sich ein Moment der Realität, des Angebrochenseins des Reiches Gottes, sondern nur insofern sie den Bericht, daß es angebrochen sei, bestätigt. Selbst dort, wo Gott selbst in einer Praxis präsent ist - wie im sakramentalen Geschehen -, ist er es immer auch verweisend, zeichenhaft, symbolisch. Insofern lautet die Frage: Verweist diese Praxis von sich aus auf die Erzählung des Heilsereignisses? Rückt sie sich selbst, indem sie dem Betroffenen ihre Bedeutung, ihren Sinn, ihre Wahrheit aufdrängt, in die Erzählung ein? Oder verweist sie deshalb auf die Erzählung, weil als darauf verweisend gedeutet werden kann bzw. weil ihr inhärierender Sinn kompatibel ist mit der Erzählung?

Die Frage nach der Wirklichkeit entstammte der spezifischen Formbestimmung der Materialität Gottes in der Verkündigung, der Erzählung. Weil sie gänzlich ungeeignet ist, Wirklichkeit entstehen zu lassen, sondern umgekehrt an die verlorene Wirklichkeit des Inhalts erinnert und selbst durch ihn konstituiert ist ${ }^{40}$, drängt die Erzählung zur Rückfrage. Aber sie wird nicht selbst zu einer konkreten Praxis, die

39 Damit stimmt auch überein, daß eigentlich nicht angemessen von einer spezifischen Form des göttlichen Wortes gesprochen werden kann. Sein entscheidendes Kennzeichen ist, daB in ihm Form und Materie überhaupt nicht mehr getrennt sind, deswegen auch nicht von einem Kompositum von Form und Materie gesprochen werden kann. Der christliche Gottesgedanke (der die integrale Wirklichkeit all dessen formuliert, was von Gott aussagbar ist) sprengt eher das Hylemorphismusmodell. Wenn menschliche Praxis genau darin, daß sie wirklich ist, am göttlichen Leben Anteil zu haben trachtet, dann hat sie, wie verborgen auch immer, auch Anteil an dem, was mit menschlicher Vernunft die Materie, die Eigenschaften Gottes sind. Also wirkliche Praxis wäre immer - anonym? - irgend wie in Zusammenhang mit göttlichem Sein.

40 Das impliziert die These, daß die Erzählung im Unterschied zu Mythen und Märchen ihren Grund nicht im isolierten subjektiven Erlebris des Erzählers hat, sondern in der externen Wirklichkeit des mitgeteilten Inhaltes. 
symbolisch in ihrer Wirklichkeit ist, sondern umgekehrt bleibt sie in der schwachen Form der Sprache universal, indem sie von der Endgültigkeit der Erlösung aller Menschen im Heilsereignis spricht. Die Verkündigung wird also nicht zur Praxis, sondern diese verweist auf sie. Genau darin, nicht selbständig zu sein und sein zu können, hat sie ihr entscheidendes Kriterium. Sie stammt aus einer Wirklichkeit, ist ihr schwacher menschlicher Abglanz und fordert, um ihre Herkünftigkeit aus einer universalen Wirklichkeit immer wieder zu rechtfertigen, wirkliche Nächstenliebe. Der Abglanz kann sich nicht verselbständigen. Wenn korrespondierende Erfahrungen ausbleiben, kann die Erzählung sie nicht ersetzen - sie kann bestenfalls zum Aushalten in der Frage, ob die Botschaft stimmt und wann denn die in ihr ausgesagte Rettung eintrete, ermutigen. Eine solche Verselbständigung der Erzählung, Substanzialisierung ihrer sprachlichen Gestalt bzw. ihre Auflösung in ein Sprachereignis ist nichts anderes als ihre Säkularisierung.

Wenn die Verkündigung eine wirkliche Praxis fordert, die sie bestätigt, dann kann dieses nicht in der Verselbständigung der Verkündigung zu einer Sprachpraxis sein. Dies ist das stärkste Argument dafür, Verkündigung und Praxis auseinanderzuhalten. Verkündigung bleibt immer angewiesen auf eine gegenwärtige punktuelle, blitzhafte Identität von Form und Materie in einem Akt der liebenden Zuwendung zum Nächsten. Darauf bleibt sie angewiesen, weil für sie ein Inhalt konstitutiv ist, der von der vergangenen universalen Befreiung berichtet. 41

Nur als solcher kann er in die Erzählung eingehen. Das Eingehen in die Erzählung von der Befreiung ist aber nicht durch seine Herkünftigkeit aus der Erzählung gewährleistet, sondern dadurch, daß es die Frage, ob denn das Heil wirklich geschichtlich erschienen sei, für einen Augenblick mit Ja beantworten läßt. Wenn diese Praxis geschieht, kann ich die Erzählung der Befreiung als universal hören, weil ich

41 Einerseits ist damit der Zirkel von Verkündigung und Glauben durchbrochen: Verkündigung bringt den Glauben nicht aus sich hervor, sondern wird umgekehrt erst verständlich und in ihrer Universalität begreifbar, wenn es ihn gibt. Sie fordert ihn, aber sie ist nicht der Glaube - vor allem nicht in seiner materiell-konkreten Realitätsdichte. Anderseits ist aber nicht jede Praxis wegen ihrer Konkretheit Hinweis auf die Wahrheit der Verkündigung, sondern nur diejenige, die durchsichtig ist, die absolute sich mitteilende Liebe. Damit läßt sich in einem ersten Schritt die Konkretheit der Glaubenspraxis denken: Sie hat immer ein natürliches Moment an sich. Sie wirkt sich in natürlichen, menschlichen Zusammenhängen aus. Sie ist eine lebensermöglichende, lebensrettende Praxis, die das gleiche biologische Substrat umfaßt wie Handlungen, die nicht oder nur schwer im Kontext der Erzählung deutbar sind. Weil sich der Glaube auf ein allgemeines biologisches Substrat des menschlichen Daseins bezieht, kann das Christentum in anderen kulturellen Kontexten und religiösen Traditionen überhaupt rezipiert und verstanden werden - ohne das vorher ein Kulturwechsel oder -zusammenbruch erfolgen muß. 
erfahren habe, daß sie auch mir gilt. So kommt meine Befreiung in der Erzählung vor.

Das hat sicherlich auch eine imperative Dimension: Nächstenliebe soll sein. Sicherlich ist in diesem Sinne Verkündigung immer auch Aufforderung an die Hörer, selbst Zeuge zu sein, selbst die Verkündigungsinhalte in ihrem Leben wirklich werden $\mathrm{zu}$ lassen und $\mathrm{zu}$ bewahrheiten, nicht nur nach korrespondierenden Erfahrungen Ausschau zu halten, sondern sie selbst zu ermöglichen. Jedoch ist das eine abgeleitete Bedeutung - die immer in der Gefahr steht, daß der Hörer der Botschaft deren Wahrheit und Bewahrheitbarkeit von seinem Willensentschluß zu handeln abhängig macht. Nur, wenn sie von Hilfsbedürftigen wirklich als Befreiung erfahren worden ist, hat sie Verweisungskraft und kann als in der Erzählung vorkommend bewußt werden. Insofern kann also die universale Wirksamkeit der Botschaft nicht von meinem Willen abhängen, sondern ich trage nur dann etwas zu ihrer Bewahrheitung bei, wenn ich tatsächlich handle. Dabei kann immer noch passieren, daß ich wohl helfen und befreien will, in Wirklichkeit aber Knechtschaft verlängere.

Mit diesem so gedachten Zusammenhang von Verkündigung und Wirklichkeit ist expressis verbis ausgeschlossen, daß die Verkündigung vom Willen eines Einzelnen oder eines geschichtsmächtigen Kollektivsubjektes abhängt, also ideologisch instrumentalisiert werden kann. Ich kann die Verkündigung nie bewahrheiten wollen. Vielmehr wird mir und anderen ihre Wahrheit einsichtig, wenn ich solidarisch gehandelt habe. Sie nur zu wollen heißt, daß mir die Entscheidung noch offen ist, sie solle nicht wahr sein. Solange ein Wille diese Möglichkeit noch hat, hat er noch nichts mit der Bestätigung der Wahrheit der Botschaft zu tun.

\subsection{Zeugnis}

Die konkrete Praxis der Solidarität ist also a) für die Beantwortung der Frage nach der Wahrheit des Evangeliums gefordert und kann b) durchsichtig auf das Evangelium sein. Ein Handeln, das sowohl diese Forderung erfüllt und tatsächlich den Blick auf die Erzählung des Heilsgeschehens lenkt, ist das Zeugnis. Darauf kann hier jedoch nur noch perspektivisch eingegangen werden. ${ }^{42}$

Die erste Schicht des Zeugnishandelns ist die Ebene der ideologischen Verneinung des Evangeliums. Die Identifizierung des Jesus von Nazareth als Sohn Gottes geriet in Widerspruch mit dem herrschenden Polytheismus Roms und vor allem mit dem Anspruch des Kaisers, selbst Gott zu sein. Von seiten der Christen mußte das Bekenntnis zu Jesus als dem Christus deswegen zum Widerspruch

42 Zum Ganzen siehe E. Arens, Bezeugen und Bekennen, Düsseldorf 1989. 
führen, weil seine Göttlichkeit, seine Messianität in klarer Kontinuität zum jüdischen Monotheismus stand. Genau dieses sich als Monotheismus artikulierende Bekenntnis zur Gottessohnschaft Jesu und die darin implizierte Verneinung weiterer Sohnschaften oder Stellvertreterschaften Gottes auf Erden brachte das Christentum in einen ideologischen Gegensatz zur "politischen Religion" ${ }^{43}$ der Weltmetropole Rom.

Wahrscheinlich hätte die römische Religion in ihrer Vielschichtigkeit und hohen Verarbeitungs- und Integrationskapazität gegenüber den Religionen eroberter und unterdrückter Völker auch den Widerspruch gegen sich tolerieren können, wenn er nur ideel gewesen wäre. Die Christenverfolgungen indizieren jedoch, daß dieser Glaube in der Substanz ein konkretes Verhalten, eine Praxis war, die die Grundplausibilitäten der römisch beherrschten Welt dadurch erschütterte, daß sie sie zu dysfunktionalen Verhaltensweisen führten - etwa den Kriegsdienst, die Sklavenfrage und anderes betreffend.

Die Konfrontation von römischer Staatsideologie und christlichem Glauben konnte aus verschiedenen Gründen sich nicht in der Konkurrenz ideologischer Apparate, von Philosophenschulen u.ä. vollziehen, sondern sie verdichtete sich in der Gerichtssituation, d.h. in der Beschuldigung einzelner, an die Definität der Erlösung in Jesus Christus zu glauben. Hier wird der Beschuldigte unter den Bedingungen der herrschenden Plausibilitäten und angesichts der Unverständlichkeit der Festlegung des Heils auf das konkrete geschichtliche Ereignis des Lebens, Sterbens und Auferstehens Jesu auf sich selbst zurückgeworfen. Die Wahrheit kann nicht durch Hinweis auf etwas anderes erwiesen, sondern nur im eigenen Verhalten verteidigt und verkündet werden im Standhalten bis zum Martyrium.

Der Glaube an den sich in Jesus Christus selbst offenbarenden einzigen Gott impliziert Verhaltensweisen, die selbst in einer ideologisch toleranten Weltherrschaft zu Widersprüchen führen mußten. Auf dieser Ebene erscheint die Zeugenschaft als Folge eines Bewußtseins, daß von den Ereignissen "in Jerusalem" wußte. Dieser sich selbst offenbarende Gott war aber genau deswegen einzig, weil sein Sohn ihn offenbart hat als derjenige, der das Heil unüberbietbar und endgültig in die Welt gebracht hat. Die ideologische Verneinung dieser Überzeugung findet dann nicht nur statt, wenn ein politischer Polytheismus als Toleranzprinzip die Erschütterungen seiner Plausibilität nicht duldet und verfolgt, sondern die Verneinung ist angesichts der Definität des Heils im Heilsereignis bereits im Leiden und Elend, in der Brutalität einer Sklavenhaltergesellschaft, in der willkürlichen Aberkennung von Lebensrechten sichtbar.

43 Siehe dazu J. Moltmann, Politische Theologie - Politische Ethik, Mainz/ München 1984, $42 f$ (Lit.). 
Diese "praktische" Verneinung der Wahrheit des Evangeliums durch effektive Aberkennung von Lebensrechten ist im 20. Jahrundert zur entscheidenden Herausforderung des Christentums geworden bzw. in der Vernichtungsmaschinerie, der gesetzmäßigen Tötung von Menschen bewußt geworden. In der systematischen Vernichtung von Menschen durch die nationalsozialistische Herrschaft gewinnt das Zeugnis seine paradigmatische Dichte im lebensrettenden Akt, der so konsequent ist, daß er durchgehalten wird bis in den eigenen Tod des Zeugen. Im Martyrium Maximilian Kolbes ist eine Praxis derart wirksam geworden. Es war eine Solidarität, die allein dem „biologischen" Überleben eines Menschen galt, unter Einsatz und Opferung des eigenen Lebens des Zeugen.

Eine solche Praxis hat den höchsten Grad an Durchsichtigkeit auf den Inhalt des Evangeliums. Sie belegt so sehr dessen Wahrheit, daß kaum unterschieden werden kann zwischen gegenwärtiger Praxis und der Erzählung des universalen Heilsereignisses. Diese Praxis des lebensrettenden Martyriums scheint durch ihr Stattfinden bereits in der Erzählung vorzukommen. Hier ist Gott so sehr mit dem gequälten Menschen verbunden, daß im Blick auf dieses Geschehen die Wahrheit seiner Botschaft unmittelbar einleuchtet. ${ }^{44}$

Jedoch ist der Familienvater, dessen Leben durch Maximilian Kolbes Märtyrertod gerettet wurde, von dieser Solidarität nicht zum ewigen Leben befreit worden. Aber die Bewahrung vor der Vernichtung im System nationalsozialistischer Konzentrationslager wird durch den extremen Verweisungscharakter des Opfertodes Kolbes zum materiellen Bild der Erlösung.

\section{Schluß}

Die bisherigen Überlegungen nähren eine gewisse Skepsis gegenüber der selbstverständlichen Gewißheit, daß die Kirche sich der technischen und kommunikativen Möglichkeiten der Werbung bedienen müsse, um ihren Verkündigungsauftrag zu erfüllen.

Einerseits scheint offensichtlich, daß Werbung die Verkündigung ihrerseits nicht bewahrheiten kann. Aber treibt sie nicht die Erzählung wenigstens die - in Bereiche menschlichen und gesellschaftlichen

44 Genau hier wäre der Ort, den Topos zu diskutieren, daß Gott selbst sein Wort bezeugt. Der Zeugnisbegriff hat seine theologische Spitze darin, daß das von ihm bezeichnete Handeln von Gott selbst vollzogen werden kann. siehe dazu Arens, Bezeugen (Anm. 42) 84ff. In einem ersten Zugriff kann dieser Topos als Deutung des extremen und paradigmatischen Glaubensvollzug, des Martyriums, verstanden werden. Der Märtyrer ruft Gott als Zeuge an, das Zeugnis Gottes selbst ist seine unvorstellbare Kraft im Standhalten, im Ertragen der Folter. 
Lebens hinein, die mit face-to-face Kommunikation, mit traditioneller Verkündigung nicht mehr erreicht werden? Ist die technisch vermittelte Präsentation der Grundbotschaft und der Grundsymbole nicht die Bedingung dafür, daß heute allerorten (und nicht nur in den abschmelzenden Ghettos geschlossener Katholizität) die Frage nach der Wahrheit gestellt werden kann - also die Erfahrungen von lebensermöglichender Solidarität im weitesten Sinne erwartet, erhofft und eingeklagt werden?

Anderseits scheint gerade die perfekte technische "Aktualisierung" der Botschaft ihre exponierteste Verselbständigung zu sein. Die sprachliche Gestalt der Verkündigung transportierte zugleich ein Bewußtsein, daß die Botschaft nur in der Gestalt des menschlichen Wortes präsent ist. Die viel perfektere bildhafte Inszenierung suggeriert zunächst größere Vollständigkeit, eine größere Adäquanz zum Inhalt bzw. eine höhrere Präsenz des Inhaltes in dem Darstellungsakt. Es scheint unsinnig, 'gegenüber massenmedialer Darstellung von Glaubensinhalten die Wahrheits- und Wirklichkeitsfrage zu stellen.

Die Überlegungen drängen dazu, einen theologischen Begriff der Werbung und der technisch vermittelten Kommunikation zu entwickeln. Dringend scheint die Frage beantwortet werden zu müssen, wie die Technik und die neuen Medien wirken. Drängen sie zu Fragen, wecken sie die Sehnsucht nach der Wirklichkeit des Dargestellten dann wäre Werbung ein Imperativ für die Kirchen - oder lassen sie diese Fragen verstummen - dann wäre Werbung ein Säkularisierungseffekt: nicht durch die Leugnung des Inhalts, nicht als seine Zurückführung auf menschliche Verhältnisse, Probleme und Sehnsüchte - so die klassische Religionskritik -, sondern durch die Universalisierung des menschlichen Maßes ihrer Form.

\section{SUMMARY: Proclamation of faith and practice}

In our times it seems that spreading of the Good News usually has to be combined with publicity. Taking this into consideration, the question of the theological meaning of preaching arises: the decisive peculiarity of preaching is based on the fact that its origin lays in God's historical acting for the salvation of mankind. Therefore, proclaiming the Christian faith is depending on the historical reality of a solidarian practice of charity and love of God. Only the experience of this twofold love to the next and to God can confirm the Good News of the promise of salvation for everybody.

As result of these considerations, the relationship between proclamation of faith and publicity cannot yet be defined clearly. These reflections urge for developing a theological theory of mass-communication which today exists only in the beginnings.

\section{RÉSUMÉ: Proclamation et pratique}

Dans la situation actuelle, l'évangélisation ne semble être d'usage que lorsque l'église a recours à la publicité. De ce fait, on se demand qu'elle est la 
signification théologique de la proclamation religieuse: une particularité importante de la proclamation consiste à ce qu'elle tire son origine du rachat historique par Dieu. C'est la raison pour laquelle la proclamation religieuse dépend de la réalité historique d'une pratique solidaire de l'amour de dieu et de son prochain. Seule l'expérience de cet amour confirme le message d'après lequel la Rédemption est promise à tous.

A partir de ces considérations - tel en est le résultat - on ne peut pas encore déterminer clairement la relation entre la proclamation et la publicité. Les considérations poussent vers une théorie théologique de la communication en masse qui n'en est qu'à ses débuts.

\section{RESUMEN: Predicación del evangelio y práctica}

La predicación del evangelio, en la situación actual, parece solamente habitual cuando la Iglesia utiliza para ello la publicidad. En este contexto surge la pregunta por el significado teológico de la predicación del evangelio por parte de la iglesia. La característica decisiva de ésta consiste en que se deriva de la actividad de salvación en un marco histórico. Por eso la prédica depende de la realidad histórica de una praxis solidaria del amor al prójimo y del amor a Dios. Sólo la experiencia de ese amor confirma el mensaje de que la salvación es para todos los hombres.

Partiendo de estas reflexiones no se puede determinar inequívocamente así es el resultado - la relación entre la predicación y la publicidad. Las reflexiones estimulan una teoría teológica de la comunicación de masas que solamente se encuentra en sus comienzos. 